\title{
Liver-Resident NK Cells: the Human Factor
}

\author{
Victoria Male (corresponding author) \\ Institute of Immunity and Transplantation \\ UCL Medical School \\ Rowland Hill Street \\ London NW3 2PF
}

Telephone: 02077940500 ex. 34064

\begin{abstract}
Mouse liver contains two NK cell populations, one of which recirculates while the other is tissue-resident. Following this discovery, a number of groups have sought to identify liverresident NK cells in humans. This essay presents an overview of recent advances in the field.
\end{abstract}

NK cells represent only a small fraction of circulating lymphocytes but account for up to $50 \%$ of the lymphocytes in the liver. Bulk liver NK cells have long been known to differ from their circulating counterparts, but only over the last five years has it become appreciated that the liver in fact contains two NK cell subsets: conventional NK cells (cNK) that circulate freely and liver-resident NK cells (IrNK).

\section{The Discovery of IrNK in Mice}

NK cells were identified in rodent liver in the mid-1970s, at roughly the same time as their discovery in the spleen. A flurry of research on splenic NK cells followed immediately, but little work was done on liver NK cells until the early years of this century. In a 2012 study that reignited interest in the topic, Gordon and colleagues observed a large population of "immature" NK cells in mouse liver, in addition to "mature" cNK [1]. On adoptive transfer, the "immature" NK cells could differentiate to cells with a "mature" phenotype. Therefore, they proposed that the two NK cell populations in the liver had a precursor-product relationship. Daussy and colleagues later repeated the adoptive transfers under more stringent conditions, providing evidence that the two populations in fact represent separate lineages [2]. Parabiosis experiments showed that one population (cNK) depends on the transcription factor Eomes and circulates freely while the other is Tbet-dependent, found in liver sinusoids and is unable to leave the liver (IrNK) [3,4]. Further interest in IrNK has been sparked by evidence that they can mediate contact hypersensitivity, and could therefore represent "memory" NK cells [3].

\section{The Hunt for IrNK in Humans}

In the light of these findings, a number of groups attempted to define IrNK in humans. CD49a is considered the definitive cell surface marker for IrNK in mice, so the first such attempt examined the expression of CD49a by human liver NK cells [5]. A small CD49a ${ }^{+}$NK cell population was found in the parenchyma and these cells are absent in peripheral, portal venous or hepatic venous blood, suggesting they are liver-resident.

NK cells in human blood are divided into CD56 bright and CD56 $6^{\text {dim }}$ subsets, where the majority are CD56 dim. Human liver is enriched in CD56 bright NK cells and these express CD69, which is now recognised as a marker not only of activation but also of tissue residence. Therefore, the next attempt at defining IrNK in humans focussed on CD56 bright NK cells, finding that they express a distinctive panel of chemokine receptors, integrins and L-selectin, which is likely to mediate their retention in the liver [6]. Like mouse IrNK, they are located primarily in the sinusoids and are likely to be retained there by the interaction of CCR5 and CXCR6, expressed by the NK cells, and their ligands on sinusoidal endothelial cells [6]. Indeed, 
another recent study has suggested that human IrNK should be defined not as CD56 bright but as $\mathrm{CXCR6}^{+}$, similar to mouse IrNK [7].

Neither CD56 nor CXCR6 expression define liver-residence, since significant CD56 bright and $\mathrm{CXCR}^{+}$populations are present in blood $[6,7]$. A consensus is now emerging that the best way to distinguish between $\mathrm{cNK}$ and IrNK in humans is by their expression of Tbet and Eomes [7-9]. Eomes ${ }^{\text {hiTbet }}{ }^{\text {to }}$ NK cells (henceforth "Eomes ${ }^{\text {hi") }}$ account for some $50 \%$ of human liver NK cells, but are completely absent in blood, and largely, but not completely, overlap with the CD56 ${ }^{\text {bright }}$ and $\mathrm{CXCR}^{+}$populations. However, the Eomes ${ }^{\text {hi }}$ population does not overlap with the CD49a+ population [5,7]. It seems, then, that there are two non-overlapping NK cell populations that are potentially liver-resident in humans: CD49a ${ }^{+} \mathrm{NK}$ cells and Eomes $^{\text {hi }}$ (largely CD56 ${ }^{\text {bright }}$ and $\mathrm{CXCR6} 6^{+}$) NK cells.

The absence of Eomes ${ }^{\text {hi }}$ NK cells in blood, together with their expression of proteins associated with tissue retention, points to these cells representing IrNK, but is not definitive. Recently, however, we have been able to carry out experiments in humans roughly equivalent to the parabiosis experiments that defined IrNK in mice. In clinical liver transplantation, donors and recipients are not routinely HLA-matched, allowing recipientderived cells to be distinguished from those originating from the donor liver by their expression of HLA variants. Taking this approach, we showed that Eomes ${ }^{\text {hi }}$ NK cells cannot exit the liver and are long-lived in the liver for up to 13 years, whereas Eomes ${ }^{10} \mathrm{cNK}$ cells recirculate freely [9]. This allows us to say with some certainty that Eomes ${ }^{\text {hi }}$ NK cells are indeed liver-resident in humans.

While performing these experiments, we made the unexpected observation that recipientderived Eomes ${ }^{\text {hi }}$ IrNK emerge within weeks of transplantation, indicating replenishment from the circulation. We further found that, on culture with cytokines that are highly expressed in the liver, sorted Eomes $^{10}$ cNK upregulate Eomes together with cell surface markers associated with an IrNK phenotype [9]. This was surprising because, in mice, cNK and IrNK are thought to form separate lineages [2]. It is, of course, possible that human and mouse IrNK differ in this respect but it is worth noting a small degree of flexibility between the two lineages in mice, even in the most stringent experiments $[2,3]$. It is also possible that the immunosuppression to which transplant patients are subjected alters the ability of circulating cells to be recruited to the liver and fill a resident niche. There is some evidence that this is the case for Kupffer cells [10] and it has been suggested that the conflicting outcomes of adoptive transfers reported by Gordon and Daussy could be a result of different conditioning regimes in the recipient mice.

\section{Future Directions}

In both mice and humans, IrNK cells are present and something of their origin and lineage is now understood. However, the function of these cells remains obscure. Since studies in humans have been informed by those in mice, experiments in mice are likely to be a necessary first step in defining the functions of IrNK in humans. Mouse IrNK may be "memory" cells [3] and CD49a+ IrNK in humans are $\mathrm{NKG}^{+} \mathrm{C}^{+}$, reminiscent of $\mathrm{NKG}^{2} \mathrm{C}^{+} \mathrm{NK}$ cells in the blood, which may have memory of HCMV [5]. Meanwhile, the longevity of the Eomes $^{\text {hi }}$ IrNK population could also be suggestive of memory [9]. Experiments to test the idea that either population represents memory cells will be challenging to perform in humans, although bulk hepatic NK cells in macaques display antigen-specific memory responses to vaccination. This supports the notion of memory NK cells in primate liver, although the subset responsible has not yet been defined [11].

Anatomical location and protein expression may also provide clues to function. CD49a+ IrNK are found in the parenchyma and express cytotoxic effector molecules and receptors for MHC class I, so it seems likely that they recognise and kill virally infected or cancerous hepatocytes. Eomes ${ }^{\text {hi }}$ IrNK, on the other hand, are found in the sinusoids and express fewer 
receptors for human targets, suggesting that they may recognise non-human cells in the blood. The liver processes blood coming from the gut, so the possibility that they may respond to bacteria or bacterial products is particularly attractive. In support of this hypothesis, mouse IrNK express high levels of AHR [3,12] while Eomes ${ }^{\text {hi }}$ IrNK in humans express IL23R and RORyt [9] and these genes are required for the development of ILC3, which are involved in the recognition of gut bacteria. Whatever the function of these cells, their frequency, longevity and conservation between mice and humans suggests that it is likely to be important and defining it will therefore represent a significant advance in our understanding of the hepatic immune system.

\section{References}

1. Gordon, S.M. et al. (2012) The transcription factors T-bet and Eomes control key checkpoints of natural killer cell maturation. Immunity 36, 55-67

2. Daussy, C. et al. (2014) T-bet and Eomes instruct the development of two distinct natural killer cell lineages in the liver and in the bone marrow. J. Exp. Med. 211, 563577.

3. Peng, H. et al. (2013) Liver-resident NK cells confer adaptive immunity in skincontact inflammation. J. Clin. Invest. 123, 1444-1456.

4. Sojka, D.K. et al. (2014) Tissue-resident natural killer (NK) cells are cell lineages distinct from thymic and conventional splenic NK cells. Elife 3, e01659.

5. Marquardt, N. et al. (2015) Cutting edge: identification and characterization of human intrahepatic CD49a+ NK cells. J. Immunol. 194, 2467-2471.

6. Hudspeth, K. et al. (2016). Human liver-resident CD56(bright)/CD16(neg) NK cells are retained within hepatic sinusoids via the engagement of CCR5 and CXCR6 pathways. J. Autoimmun. 66, 40-50

7. Stegmann, K.A. et al., (2016) CXCR6 marks a novel subset of T-bet(lo)Eomes(hi) natural killer cells residing in human liver. Sci Rep. 6, 26157

8. Harmon, C. et al. (2016) Tissue-resident Eomes(hi) T-bet(lo) CD56(bright) NK cells with reduced proinflammatory potential are enriched in the adult human liver. Eur. J. Immunol. 46, 2111-2120

9. Cuff, A.O. et al. (2016) Eomeshi NK Cells in Human Liver Are Long-Lived and Do Not Recirculate but Can Be Replenished from the Circulation. J. Immunol. 197, 42834291

10. Scott, C.L. et al. (2016) Bone marrow-derived monocytes give rise to self-renewing and fully differentiated Kupffer cells. Nat. Commun. 7, 10321

11. Reeves, R.K. et al. (2015) Antigen-specific NK cell memory in rhesus macaques. Nat. Immunol.16, 927-932.

12. Zhang, L.H. et al. (2016) The aryl hydrocarbon receptor is required for the maintenance of liver-resident natural killer cells. J Exp Med. 213, 2249-2257

13. Tang, L. et al. (2016) Differential phenotypic and functional properties of liverresident NK cells and mucosal ILC1s. J Autoimmun. 67, 29-35 
Box 1. Which of the human IrNK populations is equivalent to IrNK in mice?

$\mathrm{CD}_{49} \mathrm{a}^{+}$human IrNK, like mouse IrNK, express CD49a but differ from them in more respects than they are similar, including frequency, anatomical location and expression of receptors recognising $\mathrm{MHC}$ class I. Therefore it seems likely that these cells are not the human equivalents of IrNK in mice. Eomes ${ }^{\text {hi }}$ human IrNK also differ from mouse IrNK in a number of respects, most strikingly in their expression of Eomes. Although the transcription factor dependence of the two subsets in humans in not known, the expression pattern of Eomes and Tbet suggests they have exchanged roles between the two species. Given the homology of the two transcription factors, and the fact that their roles are often redundant, perhaps this should not be too surprising. On the other hand, Eomes ${ }^{\text {hi }}$ human IrNK and mouse IrNK do correspond in their frequency, location in the sinusoids and expression of a number of proteins, such as receptors recognising MHC class I, that are likely to be relevant to their function. Therefore, although neither of the two human IrNK populations is precisely equivalent to mouse IrNK, the Eomes ${ }^{\text {hi }}$ population seems to be the more similar of the two. The phenotypic and functional characteristics of $\mathrm{cNK}$ and IrNK in both species are summarised in Table 1. 
Table 1. Features of cNK and IrNK in mice and humans. -: not expressed; +: low expression/a small fraction of cells are positive; ++: intermediate expression/approximately half of cells are positive; +++ high expression/the majority of cells are positive; ND: not determined; N/A: not applicable. *: In humans, CD56 ${ }^{\text {bright }}$ CD16 ${ }^{-}$NK cells are considered less mature than CD56 dim CD16 ${ }^{+}$cells; in mice as NK cells mature, they first acquire CD11b (integrin $\alpha \mathrm{M}$ ) and then lose CD27 (a TNFR family molecule). ${ }^{* *}$ : KIR family in humans, Ly49 family in mice. ${ }^{* * *}$ : There are conflicting reports of granzyme expression in mouse IrNK. It has been reported that granzyme A mRNA does not differ between IrNK and cNK [4], whereas another study identified cNK as expressing granzyme A protein, whereas IrNK did not [2]. At the protein level, granzyme B has been reported to not differ between IrNK and cNK [13], or to be expressed in cNK but not IrNK [2].

\begin{tabular}{|c|c|c|c|c|c|}
\hline & $\begin{array}{l}\text { Mouse } \\
\text { cNK }\end{array}$ & $\begin{array}{l}\text { Mouse } \\
\text { IrNK }\end{array}$ & $\begin{array}{l}\text { Human } \\
\text { cNK }\end{array}$ & $\begin{array}{l}\text { Human } \\
\text { CD49a+ } \\
\text { IrNK }\end{array}$ & $\begin{array}{l}\text { Human } \\
\text { Eomeshi } \\
\text { IrNK }\end{array}$ \\
\hline Location & Circulating & Sinusoidal & Circulating & Parenchymal & Sinusoidal \\
\hline$\%$ of total NK in liver & $\mathrm{N} / \mathrm{A}$ & $\sim 50 \%$ & $\mathrm{~N} / \mathrm{A}$ & $0-12 \%$ & $\sim 50 \%$ \\
\hline \multicolumn{6}{|l|}{ Cell surface markers } \\
\hline CD49a (integrin $\alpha 1$ ) & - & + & - & + & - \\
\hline CD49b (integrin $\alpha 2$ ) & + & - & ND & ND & ND \\
\hline CD69 & - & + & - & + & + \\
\hline CD127 (IL-7 receptor) & - & + & - & - & - \\
\hline $\begin{array}{l}\text { CXCR6 (chemokine } \\
\text { receptor) }\end{array}$ & - & + & - & - & + \\
\hline Maturation markers* & ++ & + & ++ & + & + \\
\hline $\begin{array}{l}\text { MHC-I specific } \\
\text { receptors** }\end{array}$ & ++ & + & ++ & +++ & - \\
\hline $\begin{array}{l}\text { NKG2A/CD94 } \\
\text { (inhibitory NK receptor) }\end{array}$ & + & ++ & + & - & ++ \\
\hline $\begin{array}{l}\text { NKp46 (activating NK } \\
\text { receptor) }\end{array}$ & + & + & + & + & + \\
\hline \multicolumn{6}{|c|}{ Transcription factor expression } \\
\hline Eomes & ++ & + & + & + & ++ \\
\hline Tbet & ++ & ++ & ++ & ++ & + \\
\hline \multicolumn{6}{|l|}{ Function } \\
\hline Perforin & ++ & + & +++ & ++ & + \\
\hline Granzyme A & ++ & - or $++^{\star * *}$ & ++ & ++ & ND \\
\hline Granzyme B & ++ & - or $++^{* * *}$ & ++ & ++ & + \\
\hline Degranulation & ++ & + & +++ & + & +++ \\
\hline TRAIL & - & ++ & - & ND & - \\
\hline Cytotoxicity & ++ & +++ & ++ & ND & + \\
\hline IFNg & ++ & ++ & ++ & + & ++ \\
\hline TNFa & ++ & +++ & ++ & + & + \\
\hline GM-CSF & ++ & +++ & ++ & + & ++ \\
\hline References & $1-4,13$ & $1-4,13$ & $5-9$ & 5 & $6-9$ \\
\hline
\end{tabular}

